\title{
Brain Lesion Segmentation through Physical Model Estimation ${ }^{\star}$
}

\author{
Marcel Prastawa and Guido Gerig \\ Scientific Computing and Imaging Institute \\ University of Utah, Salt Lake City, UT 84112, USA \\ \{prastawa,gerig\}@sci.utah.edu
}

\begin{abstract}
Segmentations of brain lesions from Magnetic Resonance (MR) images is crucial for quantitative analysis of lesion populations in neuroimaging of neurological disorders. We propose a new method for segmenting lesions in brain MRI by inferring the underlying physical models for pathology. We use the reaction-diffusion model as our physical model, where the diffusion process is guided by real diffusion tensor fields that are obtained from Diffusion Tensor Imaging (DTI). The method performs segmentation by solving the inverse problem, where it determines the optimal parameters for the physical model that generates the observed image. We show that the proposed method can infer reasonable models for multiple sclerosis (MS) lesions and healthy MRI data. The method has potential for further extensions with different physical models or even non-physical models based on existing segmentation schemes.
\end{abstract}

\section{Introduction}

Brain lesions are loosely defined as abnormal tissue structures that are somehow damaged. Their appearance is highly correlated with many degenerative disorders, such as multiple sclerosis (MS), lupus, and stroke. Quantitative analysis of brain lesions, as observed through MRI, is becoming an important part for clinical diagnosis, planning of treatment, and evaluation of drug efficacy. Fully automatic lesion segmentation methods that are objective and reproducible are necessary to facilitate the study of large population studies involving brain lesions.

Automatic segmentation of lesions from MRI data is a challenging problem. Lesions typically have weakly defined borders against the surrounding structures (which can be white matter by itself, or both white and gray matter). The shapes of the individual lesions generally follow the definition of the fiber tracts in white matter. The FLAIR (Fluid-Attenuated Inversion Recovery) channel, the typical MRI modality used for lesion detection, is almost always corrupted with an

\footnotetext{
* This work is part of the National Alliance for Medical Image Computing (NA-MIC), funded by the National Institutes of Health through Grant U54 EB005149. Information on the National Centers for Biomedical Computing can be obtained from http://nihroadmap.nih.gov/bioinformatics
}

G. Bebis et al. (Eds.): ISVC 2008, Part I, LNCS 5358, pp. 562 571, 2008.

(C) Springer-Verlag Berlin Heidelberg 2008 
artifact where the ventricle boundaries appear bright. This artifact is caused by the combination of the pulsation of the ventricles and the long echo time (TE) for the FLAIR protocol [1. It is difficult to account for all these problems in lesion segmentation schemes without using a good model of the underlying physical process or some empirical rules.

Many methods have been proposed for automatic lesion segmentation, particularly for MS lesions. Zijdenbos et al. proposed a method based on a neural network classifier [2]. Thirion et al. [3] and Rey et al. 4] segmented lesions based on the local deformation between scans at different time points. van Leemput et al. [5] proposed a method that detects lesions as outliers from the intensity distributions of healthy tissue. However, most of the lesion segmentation schemes do not use physical models of pathology. Physical modeling has been successfully applied for solving the registration problem for pathology. Mohamed et al. 6] used a tumor growth model to estimate the deformation map between a healthy subject and a tumor subject. The registration is driven by manually selected landmarks within the brain.

We propose a method for segmenting lesions from brain MRI by estimating the underlying physical model. The physical model used in this paper is the classic reaction-diffusion process, where the diffusion process is guided by a diffusion tensor field [7]. Each diffusion tensor describes the likely diffusion directions of water within a local region. Segmentation is performed by determining the parameters for the physical model that best matches the observed images. We demonstrate the results of applying the method on an multiple sclerosis (MS) lesion dataset and a healthy dataset. In the MS lesion data, we show that the inferred model for lesions generates images that are similar to the observed MR images. In the healthy data, we show that the method correctly infers that the lesion model is inappropriate. Our method is not only useful for segmenting the observed lesions in the MR images, as it also estimates the underlying lesion formation process which may have potential for further analysis.

\section{Method}

The method computes a model for the observed multimodal $\mathrm{MR}$ images $I_{O}=$ $\left\{I_{O}^{c}\right\}$ where $c$ indexes the different modalities (in this paper $\mathrm{T} 1 \mathrm{w}$ and $\mathrm{T} 2 \mathrm{w}$ ). The model $M$ is composed of two components: a physical model for pathology, and an image generation model. Both models uses the information contained in the template data, shown in Figure 1. The template data contains information about the expected MR intensities, the anatomical structure (in the form of a set of spatial priors), and the expected diffusion tensor field. We use the MR images and anatomical information provided by the Montreal Neurological Institute (MNI) through the BrainWeb interface [8]. The BrainWeb data does not contain the diffusion tensor image information, so we align mean diffusion tensor of a separate population to the BrainWeb data. The diffusion tensor field for the population are created using the method described by Goodlett et al. [9] 

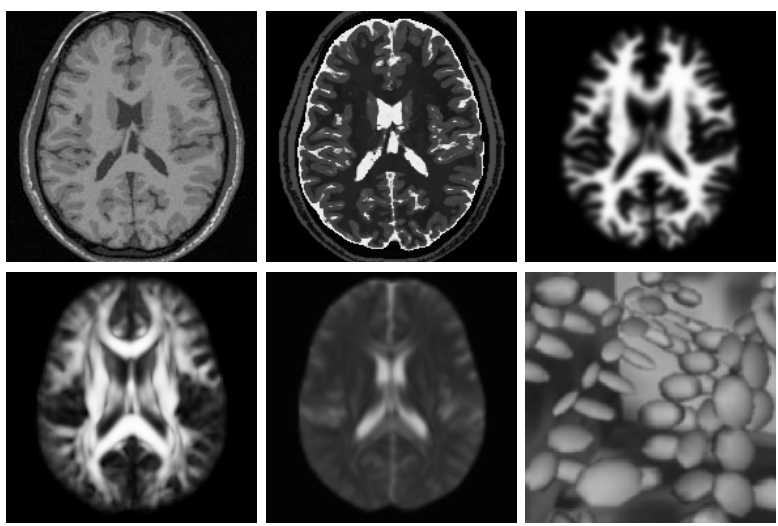

Fig. 1. The template data used for inferring the physical model and the image generation model. The model is based on subject 04 from the twenty normals BrainWeb database. Top row, from left to right: axial view of the $\mathrm{T} 1 \mathrm{w}$ image, $\mathrm{T} 2 \mathrm{w}$ image, and the white matter prior. Bottom row, from left to right: the fractional anisotropy (FA) values that show the structure of the fibers as observed through the diffusion tensors, the mean diffusivity (MD) values that show the magnitude of the diffusion tensor field, and a $3 \mathrm{D}$ view of a portion of the tensor field where each tensor is represented by an ellipsoid.

All image alignment or registration are performed using affine transformations and deformable transformations parametrized using B-splines with the mutual information image match metric [10].

\subsection{Physical and Image Models}

We assume that the lesion formation process is modulated by the underlying fiber structure, so the pathological process of lesions is modeled using the following reaction-diffusion equation:

$$
\frac{\partial \phi}{\partial t}=-\nabla \cdot(\alpha D \nabla \phi)+\beta \phi
$$

where $\phi(x, t)$ denotes the lesion probability at location $x$ and time $t, D$ denotes the diffusion tensor field, $\alpha$ denotes the diffusion modifier, and $\beta$ denotes the reaction coefficient. In this model, $\alpha$ is a scalar that represents the rate of spread for lesions and $\beta$ is a scalar that represents the local growth rate for lesions. The solution for $\phi$ is obtained by discretization and interpolation using the finite element method 11.

As part of the physical model, we also specify the points where lesions begin to appear. The set of $n$ lesion seed points are denoted by $X_{\text {seeds }}=\left\{x_{k} \mid 1 \leq k \leq n\right\}$. Each individual lesion seed point $x_{i}$ is used to define an initial local probability 
$\phi^{(i)}$ that is evolved independently with different parameters $\alpha_{i}$ and $\beta_{i}$. The initial lesion probability $\phi^{(i)}(x, t=0)$ is defined using the following equation:

$$
\phi^{(i)}(x, t=0)= \begin{cases}1 & \text { if }\left|x-x_{i}\right|<\epsilon \\ 0 & \text { otherwise. }\end{cases}
$$

The final lesion probability is the accumulation of the probabilities associated with each seed point after applying the reaction-diffusion process:

$$
p_{\text {lesion }}(x)=\sum_{i} \phi^{(i)}\left(x, t=t_{s}\right)
$$

where $t_{s}$ is the simulation time.

In addition to the physical model for pathology, we also model the appearance of MRI presenting lesions. We synthesize MR images $I_{M}$ from the physical model $M$ that is constrained by Equation 1, More precisely, given the lesion probability field $\phi$, the synthesized MR images are computed using the following equation.

$$
I_{M}^{c}(x)=\left(1-p_{\text {lesion }}(x)\right) \times I_{T}^{c}(x)+p_{\text {lesion }}(x) \times \mathcal{L}^{c}
$$

where $I_{T}$ is the set of MRI images from the template, and $\mathcal{L}^{c}$ is the expected lesion appearance for channel $c$. We assume that the simulation time $t_{s}$ is known or can be reasonably approximated. The lesion appearance in each channel is computed as follows:

$$
\mathcal{L}^{c}=I_{T}^{w m}+\operatorname{range}\left(I_{T}\right) \times \mathbb{E}_{x \in \text { lesion }}\left[\frac{\left(J(x)-J^{w m}\right)}{\operatorname{range}(J)}\right]
$$

where $I_{T}^{w m}$ is the white matter intensity for the template, $\mathbb{E}[]$ denotes the expectation function, $J$ is a training image with marked lesion regions, and $J^{w m}$ is the white matter intensity for the training image $J$.

We formulate the segmentation problem as an inverse problem. Given the observed image $I_{O}$, segmentation is equivalent to determining the optimal parameters for the reaction-diffusion equation (Equation 1) that generates the synthetic image $I_{M}$ that best matches the observation. The image match function in our algorithm is the linear combination of the mutual information image match metric, which is chosen as MR images are not normalized or standardized across different scans:

$$
\operatorname{match}\left(I_{O}, I_{M}\right)=\sum_{c} w_{c} \mathcal{M I}\left(I_{O}^{c}, I_{M}^{c}\right)
$$

where each $w_{c}$ is a predefined weight for channel $c$ that determines the relative influence of each channel in the estimation. The mutual information for images with modality $c$ is the information-theoretic measure

$$
\mathcal{M I}\left(I_{O}^{c}, I_{M}^{c}\right)=\mathcal{H}\left(I_{O}^{c}\right)+\mathcal{H}\left(I_{M}^{c}\right)-\mathcal{H}\left(I_{O}^{c}, I_{M}^{c}\right)
$$

where $\mathcal{H}(\cdot)$ denotes entropy. The mutual information image match metric has been successfully used for aligning 3D medical images [10. 


\subsection{Optimization Scheme}

The optimization scheme for match $\left(I_{O}, I_{M}\right)$ starts with an initial definition of a set of seed points. The points can be drawn at random or chosen using some heuristics to obtain most likely candidates. In this paper, the initial selection of lesion seed points $X_{\text {seeds }}$ is performed by first partitioning the image into relevant regions by applying the watershed transform to the magnitude of the multivariate gradient [12. We then select the region centers that have high white matter probability and $\mathrm{T} 2 \mathrm{w}$ intensity larger than the expected gray matter $\mathrm{T} 2 \mathrm{w}$ intensity. More precisely, after partitioning $I_{O}$ into a set of regions $R_{i}$ where $R_{i} \cap R_{j}=0, \forall i \neq j$, we pick locations $\bar{x}_{i}=\frac{1}{\left|R_{i}\right|} \sum_{x \in R_{i}} x$ as lesion seed points where

$$
\left[\frac{1}{\left|R_{i}\right|} \sum_{x \in R_{i}} p(x \mid w m)\right]>0.9 \text { and }\left[\frac{1}{\left|R_{i}\right|} \sum_{x \in R_{i}} I_{O}^{(T 2)}(x)\right]>\operatorname{median}\left(I_{O}^{(T 2)} \mid g m\right) .
$$

This condition ensures that the method chooses the centers of regions with high white matter probability and are brighter than the median of the $\mathrm{T} 2 \mathrm{w}$ image samples obtained by sampling the prior $p(x \mid g m)$. The condition associated with the $\mathrm{T} 2 \mathrm{w}$ image channel is used to exclude samples that are unlikely to be lesions for efficiency. However, the method can proceed without enforcing the T2w intensity constraint.

Due to the combinatorial cost in computing the model parameter $M$ with the optimal image match measure, we propose the following greedy algorithm:

1. Initialization: Set $X_{\text {seeds }}=\left\{\bar{x}_{i}\right\}$, following the condition in Equation 8 Initialize $I_{M}$ using template image intensities, and initialize the lesion probability $p_{\text {lesion }}(x) \leftarrow 0$.

2. Seed insertions: For each seed point $x_{i} \in X_{\text {seeds }}$, the algorithm attempts to insert a single lesion generated by evolving $\phi^{(i)}\left(x_{i}, 0\right)=1$ following Equation 1. Each point $x_{i}$ is associated with different reaction-diffusion parameters $\alpha_{i}$ and $\beta_{i}$, which are initialized by setting $\alpha_{i}=1$ and $\beta_{i}=0.5$.

(a) Position update: The point $x_{i}$ is perturbed using Brownian motion to determine the optimal insertion location in the local neighborhood

(b) Model parameter update: Optimize $\alpha_{i}$ and $\beta_{i}$ so that they maximize the image match function match $\left(I_{O} \mid I_{M}\right)$. $I_{M}$ is generated using the global lesion probability combined with the probability $\phi^{(i)}(x, t)$ generated by evolving the probability defined around the lesion seed point $x_{i}$ using the reaction-diffusion process governed by $\alpha_{i}$ and $\beta_{i}$.

(c) Global update: If the insertion of point $x_{i}$ with optimal parameters $\alpha_{i}$ and $\beta_{i}$ results in the increase of match $\left(I_{O}, I_{M}\right)$, the algorithm updates the global lesion probability:

$$
p_{\text {lesion }}(x) \leftarrow p_{\text {lesion }}(x)+\phi^{(i)}(x, t) .
$$

The algorithm also updates the synthetic images $I_{M}$ by applying Equation 4. Otherwise, the point $x_{i}$ is rejected and the global lesion probability and $I_{M}$ are unchanged. 


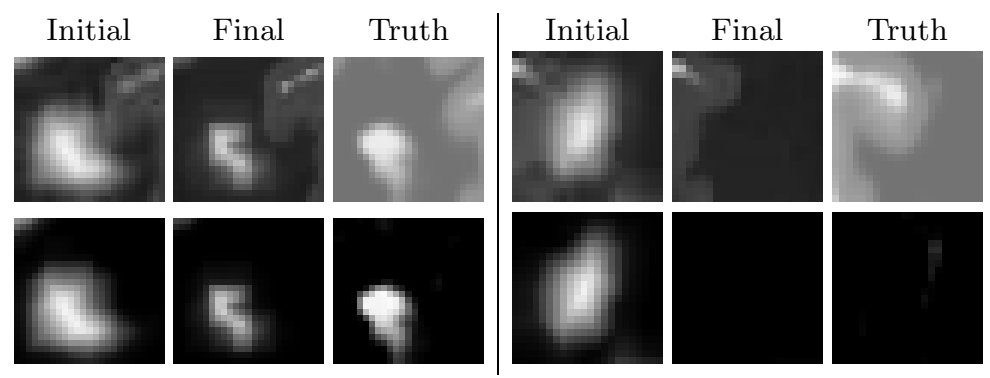

Fig. 2. Behavior of the algorithm for different initial seed points. Left block: a proper seed point that is inserted into the final model, the lesion model parameters have been optimized to fit the observed image. Right block: an incorrect seed point that is rejected by the algorithm. Top row: the $\mathrm{T} 2 \mathrm{w}$ image intensities. Bottom row: the lesion probabilities.

Figure 2 shows the result of the seed insertions and the optimizations for the local reaction-diffusion parameters for a single lesion seed point, where an incorrect seed point is not inserted and the the underlying lesion probability from a correct seed point becomes more sharply defined. The seed point is rejected due to the reduced image match and the lesion probabilities in the region are unchanged for the case where we have an improper seed point. The lesion appearance is optimized to fit the observed data for the case where we have a correct seed point.

\section{Results}

We have applied the method to two datasets. The first dataset is the MS lesion BrainWeb MRI, and the second dataset is the original healthy BrainWeb MRI. Figure 3 show the input MR images, the synthesized MR images, and the lesion probability generated by the method.

The ground truth segmentations are compared against the computed lesion segmentations using a volumetric measure and a spatial overlap measure. The volumetric measure is the total lesion load (TLL), which is computed as the sum of the lesion probabilities: $\sum_{x} p($ lesion $\mid x)$. The spatial overlap measure is the Dice similarity coefficient (DSC), which is defined for two binary segmentations $A$ and $B$ as the ratio between the volume of overlap regions and the average volume:

$$
D S C(A, B)=2 \frac{|A \cap B|}{|A|+|B|} .
$$

We compute the lesion binary mask by thresholding the lesion probabilities, where probability values $>0.5$ is considered a lesion object. The DSC overlap metric tend to assign higher penalty for errors in the segmentation of small structures. A good segmentation of a large brain structure such as the white matter typically yield a DSC value above $90 \%$. 
For the BrainWeb MS lesion data, the ground truth TLL is 6697.5 and the TLL for the probability generated by our method is 5395.3. This shows a difference of approximately $20 \%$ compared to the ground truth TLL. The amount of spatial overlap between the automatic segmentation results and the ground truth is measured to be $64 \%$. The DSC value for our lesion segmentation is lower than the ideal value of $90 \%$ due to the fact that lesions typically appear as multiple small, spatially disconnected structures. Given this spatial configuration, the

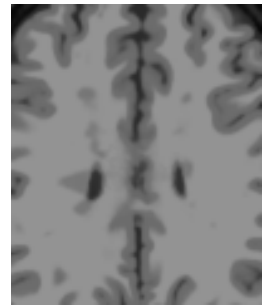

(a)

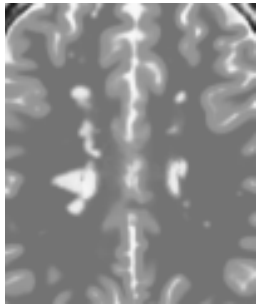

(b)

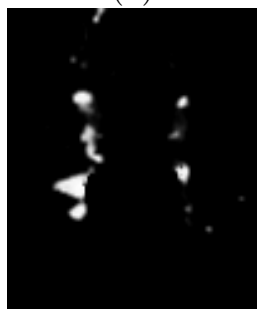

(e)

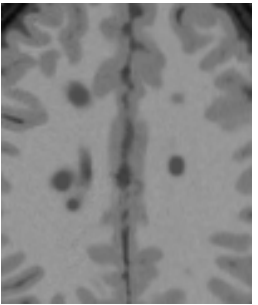

(c)

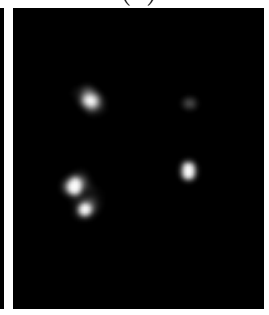

(f)

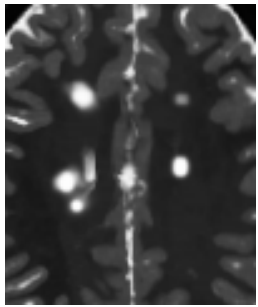

(d)

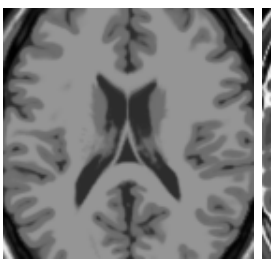

(a)

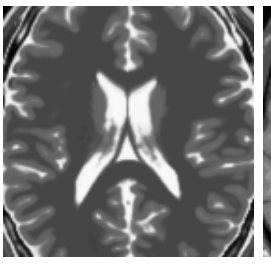

(b)

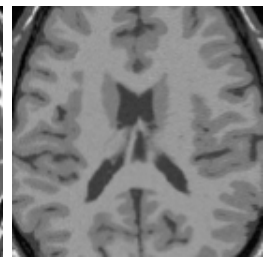

(c)

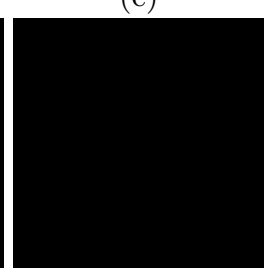

(f)

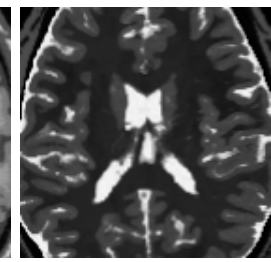

(d)

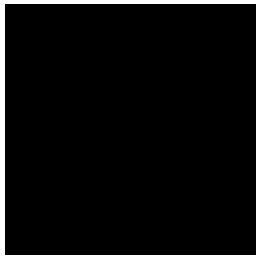

(e)

Fig. 3. Results of the method proposed to this paper for the BrainWeb MS lesion data (top) and the original healthy BrainWeb data (bottom). The individual images for each row are: axial views of the (a) input T1w image, (b) the input T2w image, (c) the synthesized T1w image, (d) the synthesized T2w image, (e) the lesion ground truth, and (f) the computed lesion probability. 
DSC measure tends to accumulate penalties for the segmentation mismatches of each small lesion object.

For the healthy BrainWeb data, the method correctly rejected all seed points and generated zero lesion probabilities. Our optimization scheme was able to determine that including anatomical changes due to lesion will result in a dataset that does not match the healthy subject dataset.

\section{Discussion}

This paper presents a new approach for segmenting lesions from multimodal brain MR images, where segmentation is performed by inferring the physical model for the underlying pathology. Compared to other lesion segmentation methods, our approach provides an estimate of the underlying lesion formation process in addition to the segmentation of lesions in MRI. Preliminary results show that the method is promising for automatic lesion detection. Our method generates 3D segmentation results, as shown in Figure 4. Quantification and localization of brain lesions in 3D might help clinicians to assess status and progress of the disease, and eventual drug efficacy as observed through white matter changes in the brain. Our approach can be generalized to more sophisticated physical and image models. One advantage of our method is that it computes the growth parameters $\alpha_{i}$ and $\beta_{i}$ for each lesion seed point in addition to the segmentation. This may have potential applications in longitudinal analysis of lesion image data.

The results demonstrate that our method has the potential of being applied to mixed populations of subjects with and without lesions. The accuracy of the lesion segmentation is limited since we have not incorporated information from

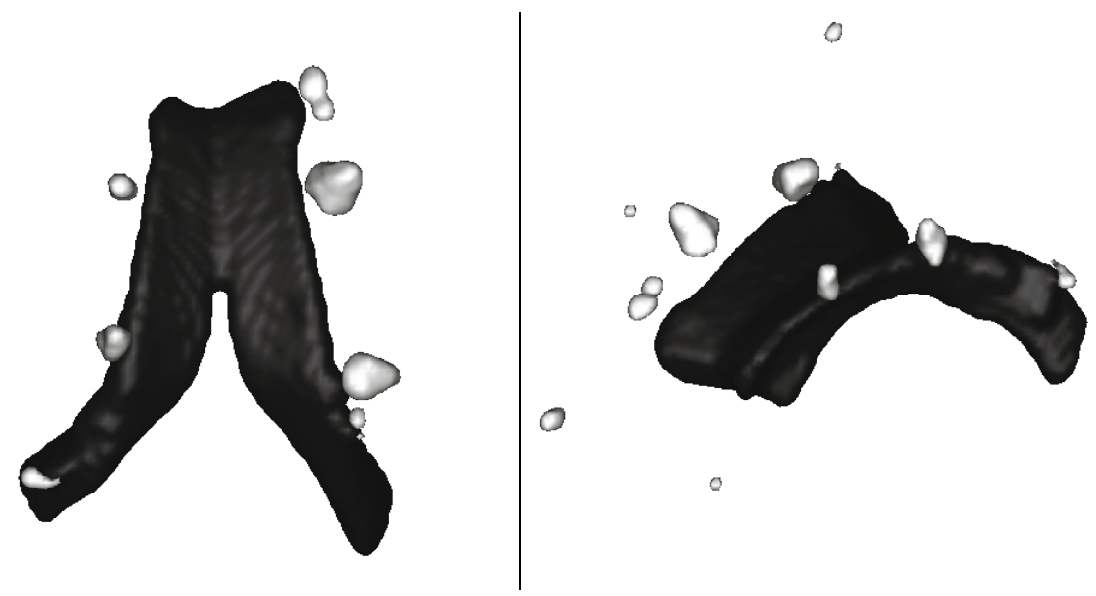

Fig. 4. 3-D views of the segmented structures from the BrainWeb MS images. Lesions are shown as the bright gray structures, and the lateral ventricles are shown in dark gray for anatomical reference. 
the subject-specific diffusion tensor fields. The mismatch of the diffusion tensor information between the DTI atlas (i.e., the population model) and the actual diffusion properties of the subject may result in suboptimal estimation of the lesion probabilities. We also detect false positives and false negatives, as shown in Figure 3. We expect that future improvements of the greedy optimization scheme, combined with a better strategy for selecting the initial seed points, will reduce the number of false positives and false negatives.

In the future, we intend to extend the method in several ways. For example, we plan to use extended physical models with a deformation model. Another extension to the model would be to determine the optimal reaction and diffusion coefficients $\alpha$ and $\beta$ based on spatial location (e.g., as defined by an anatomical parcellation). We also plan to incorporate the FLAIR image channel by modeling the pulsation of the ventricular system. The current model can also be extended by incorporating longitudinal information. In particular, the segmentation of a previous time point can be used to drive the segmentation of a different time point 34]. We currently use high resolution diffusion tensor information by averaging over a population. To improve the accuracy of our segmentations, we will need to incorporate the subject specific diffusion tensor information when they are available.

The models used in this segmentation framework need not be restricted to physical models. We plan to explore the combination of statistical segmentation schemes and machine learning models into the framework.

\section{References}

1. Bakshi, R., Caruthersa, S.D., Janardhana, V., Wasay, M.: Intraventricular csf pulsation artifact on fast fluid-attenuated inversion-recovery MR images: Analysis of 100 consecutive normal studies. AJNR 21, 503-508 (2000)

2. Zijdenbos, A., Forghani, R., Evans, A.: Automatic quantification of MS lesions in 3D MRI brain data sets: Validation of INSECT. In: Wells, W.M., Colchester, A.C.F., Delp, S.L. (eds.) MICCAI 1998. LNCS, vol. 1496, pp. 439-448. Springer, Heidelberg (1998)

3. Thirion, J.P., Calmon, G.: Deformation analysis to detect and quantify active lesions in three-dimensional medical image sequences. IEEE TMI 18, 429-441 (1999)

4. Rey, D., Subsol, G., Delingette, H., Ayache, N.: Automatic detection and segmentation of evolving processes in 3D medical images: Application to multiple sclerosis. Medical Image Analysis 6, 163-179 (2002)

5. van Leemput, K., Maes, F., Vandermeulen, D., Colchester, A., Suetens, P.: Automated segmentation of multiple sclerosis lesions by model outlier detection. IEEE TMI 20, 677-688 (2001)

6. Mohamed, A., Zacharaki, E.I., Shen, D., Davatzikos, C.: Deformable registration of brain tumor images via a statistical model of tumor-induced deformation. MedI.A 10 (2006)

7. Clatz, O., Sermesant, M., Bondiau, P.Y., Delingette, H., Warfield, S.K., Malandain, G., Ayache, N.: Realistic simulation of the $3 \mathrm{~d}$ growth of brain tumors in mr images including diffusion and mass effect. IEEE Transactions on Medical Imaging 24, 1344-1346 (2005) 
8. Cocosco, C.A., Kollokian, V., Kwan, R.S., Evans, A.C.: BrainWeb: Online interface to a 3D MRI simulated brain database. Neuroimage 5 (1997)

9. Goodlett, C., Davis, B., Jean, R., Gilmore, J., Gerig, G.: Improved correspondence for dti population studies via unbiased atlas building. In: Larsen, R., Nielsen, M., Sporring, J. (eds.) MICCAI 2006. LNCS, vol. 4191, pp. 260-267. Springer, Heidelberg (2006)

10. Maes, F., Collignon, A., Vandermeulen, D., Marchal, G., Suetens, P.: Multimodality image registration by maximization of mutual information. IEEE Trans. Med. Imaging 16, 187-198 (1997)

11. Hughes, T.J.R.: The finite element method: linear static and dynamic finite element analysis. Dover (2000)

12. Lee, H.C., Cok, D.R.: Detecting boundaries in a vector field. IEEE Trans. Signal Processing 39, 1181-1194 (1991) 